Z. Klin. Chem. Klin. Biochem.

12. Jg. 1974, S. 14-22

\title{
Berechnungen über die Wirtschaftlichkeit von mechanisierten Analysensystemen. Ein Vorschlag zur Schätzung der kritischen Serienlänge
}

\author{
Von R. Haeckel, P. Höpfel und G. Höner \\ Institut für Klinische Chemie der Medizinischen Hochschule Hannover
}

(Eingegangen am 28. September 1973)

Die Rentabilitätsschwelle zwischen manuellen und mechanisierten Verfahren wird als kritische Serienlänge bezeichnet: darunter wird die serielle Probenanzahl pro Arbeitstag verstanden, bei der die Gesamtkosten einer Analy senserie für ein manuelles und für ein mechanisiertes Verfahren gleich hoch sind.

Da die Gesamtkosten (fixe + variable Kosten) einer Analysenserie linear mit der Probenanzahl ansteigen, läßt sich eine einfache Formel zur Berechnung der kritischen Serienlänge ableiten, deren Anwendbarkeit an verschiedenen Beispielen demonstriert wird. Außerdem wird gezeigt, daß die kritische Serienlänge eines indiskriminierenden Mehrkanalgerätes von 35 auf 61 (280) Proben ansteigt, wenn von 6 obligatorisch durchgeführten Analysen nur 5 (3) indiziert sind.

\section{Determination of the economy of mechanized analytical systems. The evaluation of the critical length of a series}

The critical length of a series is used to denote the profitability threshold between manual and mechanized methods, and it is defined as the number of serial samples per working day for which the total cost is the same for both manual and mechanized methods.

Since the total costs (fixed + variable) of a serial analy sis increase linearly with the number of samples, it is possible to derive a simple formula for the calculation of the critical length of a series. Various examples are given for the application of this formula. In addition, it is shown that the critical length of a series for an indiscriminate multichannel apparatus increases from 35 to 61 (or 280 ) samples, if only 5 (or 3 ) out of 6 obligatory analyses are recorded.

Das Angebot der Industrie an mechanisierten Analysensystemen kann in seiner Breite nur noch von Fachleuten überschaut werden. Einer der wesentlichen Faktoren, die die Anschaffung von Analysengeräten begrenzen, sind die Gesamtkosten (1). Da der Käufer meist nur den Kaufpreis kennt, aber keine Daten über die genauen Betriebskosten zur Verfügung hat, kann er sich im allgemeinen keinen Überblick über die Rentabilität der Geräte verschaffen. Trotzdem wird in der Laienpresse viel über eine angebliche Verbilligung der Analysen beim Einsatz von Analysenautomaten geschrieben.

Untersuchungen über die Wirtschaftlichkeit der verschiedenen Analysensysteme scheinen dringend erforderlich zu sein. Vor kurzem haben wir ein einfaches Verfahren vorgeschlagen, das auf der Abschätzung der sogenannten kritischen Serienlänge beruht (1). Diese vermittelt einen Richtwert, die dem Anwender von Analysengeräten anzeigt, bei welcher Probenfrequenz sich die Mechanisierung eines Meßplatzes lohnt.

Im Folgenden wird die Anwendung dieses Verfahrens zur Ermittlung der kritischen Serienlänge für verschiedene Analysensysteme, sowie die Wirtschaftlichkeit von indiskriminierten Mehrfachanalysen untersucht.

\section{Die kritische Serienlänge, Definition und Ermittlung}

Als kritische Serienlänge wird die Probenanzahl bezeichnet, die an einem Arbeitstag als Serie mindestens anfallen muß, damit die Gerätekosten im Vergleich zu den entsprechenden manuellen Verfahren durch die eingesparten laufenden Kosten gerade ausgeglichen werden (Tab. 1). Die Berechnung einer kritischen Serienlänge ist demnach nur dann möglich, wenn die laufenden Kosten des mechanisierten Verfahrens geringer als bei der entsprechenden manuellen Vergleichsmethode sind. Bei der Ermittlung der Probenanzahl werden erforderliche Standard-, Leerwert- und Kontrollproben nicht mitgezählt; sie werden jedoch bei der Analysenzeit mit bericksichtigt.

Im Folgenden wird zwischen fixen (für Geräte, Wartung, Glaswaren) und variablen Kosten (für Reagenzien, Einmalmaterial, Personal) unterschieden.

\section{Die fixen Kosten}

Die Kosten für die Anschaffung der Geräte entsprechen den Listenpreisen der Hersteller (einschl. der Mehrwertsteuer von $11 \%$ ). Glaswaren verschwinden immer mehr . aus dem medizinischen Labor zugunsten von Einmalmaterialien. Wenn Volumina über $0,5 \mathrm{ml}$ pipettiert werden müssen, werden Vollpipetten (Blaubrand) eingesetzt und die Preise dem Brand-Katalog 1972 (K. Brand, D-698 Wertheim) entnommen. Für Vollpipetten und Glasküvetten (Hellma GmbH., D-784 Mülheim) wird eine Lebensdauer von 100 Arbeitstagen angenommen. Problematisch sind die anzusetzenden Kosten für die Wartung der Geräte. Hierzu wurden bisher nur wenige 


\section{Tab. 1. Die Bestimmung der kritischen Serienlänge}

Bei der kritischen Serienlänge $\left(S_{k}\right)$ sind die Gesamtkosten $\left(K_{t}\right)$ einer Analysenserie für $A$ (manuelle Vergleichsmethode) und B (mechanisiertes Verfahren) gleich:

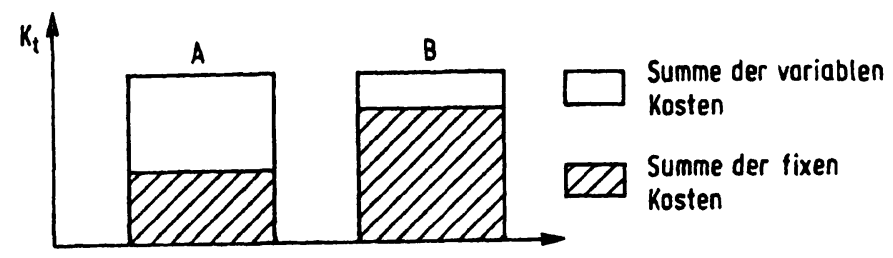

Fixe Kosten pro Arbeitstag $\left(\mathrm{K}_{\mathrm{f}}\right)$ :

$$
K_{f}=\frac{L\left(1+\frac{S}{100} \cdot T_{1}\right)}{T_{2} \cdot T_{3}}+\frac{G}{T_{4}}+E_{f}+R_{f}
$$

L = Listenpreis des Gerätes

$S=$ Servicekosten pro Jahr in Prozent von L (z. B. 1,5)

$T_{1}=T_{2}$ minus Garantiezeit in Jahren (meistens $T_{2}-1$ )

$T_{2}=$ Voraussichtlicher zeitlicher Einsatz des Gerätes in Jahren (z. B. 6 Jahre)

$T_{3}=$ Anzahl der Arbeitstage pro Jahr (z. B. 300 für 1972)

$\mathbf{G}=$ Kosten für Glaswaren

$T_{4}=$ Geschätzte Haltbarkeit für die verwendeten Glaswaren in Tagen (z. B. 100 Tage)

$E_{\mathbf{f}}=$ fixe Kosten für Einmalmaterialien pro Serie

$\mathbf{R}_{\mathbf{f}}=$ fixe Kosten für Reagentien pro Serie

Variable Kosten pro Analysenserie $\left(\mathrm{K}_{\mathrm{v}}\right)$ :

$$
K_{v}=\left(E_{p}+R_{p}\right) \cdot n+P \cdot t_{n}
$$

$\mathbf{E}_{\mathbf{p}}=$ Einmalmaterial pro Probe

$\mathbf{R}_{p}=$ Reagenzienkosten pro Probe

$\mathbf{P}=$ Personalkosten pro Minute (z. B.: 0,22 DM)

$t_{n}=$ Analysenzeit (Minuten für eine Serie von $n$ Proben

Gesamtkosten einer Analysenserie:

$$
K_{t}=K_{f}+K_{v}
$$

Kritische Serienlänge:

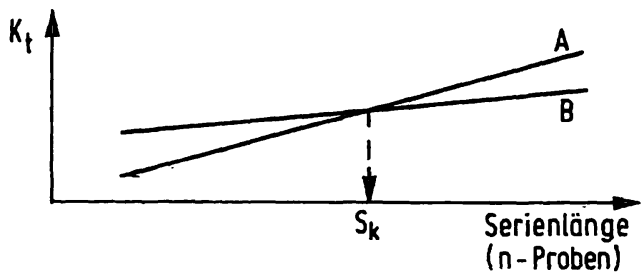

Daten veröffentlicht. Daher sind vorerst bestimmte Annahmen unumgänglich. Vor kurzem (1) haben wir eine Zusammenstellung der uns 1972 entstandenen Servicekosten (einschließlich der Telefonkosten) für vẹrschiedene Analysenautomaten mitgeteilt. Alle Geräte befanden sich im 1. oder 2. Jahr nach der abgelaufenen Garantiezeit. Im folgendem Jahr betrugen die Servicekosten an den gleichen Geräten 2,7\% (in Vorbereitung); da sie in späteren Jahren noch etwas ansteigen werden, wird der durchschnittliche Prozentsatz für die gesamte Nutzungsdauer zwischen 3 und $5 \%$ liegen, je nach technischem Geschick des Anwenders.
Die fixen Gerätekosten werden für einen Arbeitstag berechnet. 1972 fielen 300 Arbeltstage an (Sonn- u. arbeitsfreie Feiertage ausgeschlossen). Außer den Gerätekosten enthalten die fixen Kosten auch alle Kosten für Einmalmaterialien und Reagenzien, die pro Serie konstant unabhängig von der Probenanzahl anfallen (z. B. für Leer- und Standardwerte, Kontrollproben etc.). Bei Analysenautomaten mit kleinen, fixierten Unterserien (z. B. Küvettenrotorverfahren) können diese Kosten auch den laufenden (= variablen) Kosten zugerechnet werden.

\section{Die variablen Kosten}

Die Kosten für Einmalmaterialien werden der Preisliste der Firma Sarstedt (W. Sarstedt, D-5223 Nümbrecht) beim Bezug von 10000 Stück entnommen. Wenn ein Analysenautomat im Mikrolitermaßstab arbeitet, sollte der Vergleich ebenfalls mit einer manuellen Mikromethode erfolgen, damit ein niedriger Reagenzienverbrauch nicht eine günstigere Rentabilität vortäuscht. Läßt sich die manuelle Vergleichsmethode jedoch aus technischen Gründen nur im Milliliterbereich durchführen, ist der Einsatz einer entsprechend hohen Reagenzienmenge für Rentabilitätsberechnungen gerechtfertigt. Diese Uberlegungen gelten insbesondere, wenn teure Reagenzien verwendet werden.

Unsere Berechnungen berücksichtigen die für Leerwert-, Standard- und Kontrollbestimmungen benötigten Reagenzien. Der Preis für die Kontrollproben selbst wird nicht eingesetzt, jedoch der für evtl. erforderliche Referenzproben, die zur Kalibrierung dienen (z. B. bei Mehrkanalgeräten). Beim Einsatz von käuflichen Testkombinationen (Reagenzienkits) haben wir zur Berechnung der kritischen Serienlänge $S_{k}$ nur eine Packungsgröße für alle Serienlängen verwendet. Dadurch werden vorerst noch gewisse Vereinfachungen vorgenommen, die zu geringen Ungenauigkeiten führen können.

Den Personalkosten (0,22 DM pro Minute) sind die in Tabelle 2 zusammengestellten Daten zugrunde gelegt. Die Personalkosten ergeben sich durch Multiplikation von 0,22 mit der pro Serie erforderlichen Analysenzeit in Minuten.

Die Analysenzeit nimmt meistens mit steigender Probenfrequenz (Abb. 1) ab. Die in Abbildung 1 dargestellte Kurve besteht aus zwei Teilen; sie fällt zunächst rasch ab und geht bei höherer Analysenfrequenz in einen asymptotischen Verlauf über. Der Knickpunkt entspricht im allgemeinen der in der 2 . Stunde möglichen Analysenfrequenz.

In grober Annäherung kann daher zur raschen Abschätzung der kritischen Serienlänge die Analysenzeit auf die in der 2. Stunde erreichbare Probenfrequenz bezogen werden (1). Die exakte Berechnung der laufenden Kosten muß jedoch unterschiedliche Analysenzeiten für verschiedene Probenfrequenzen berücksichtigen. 


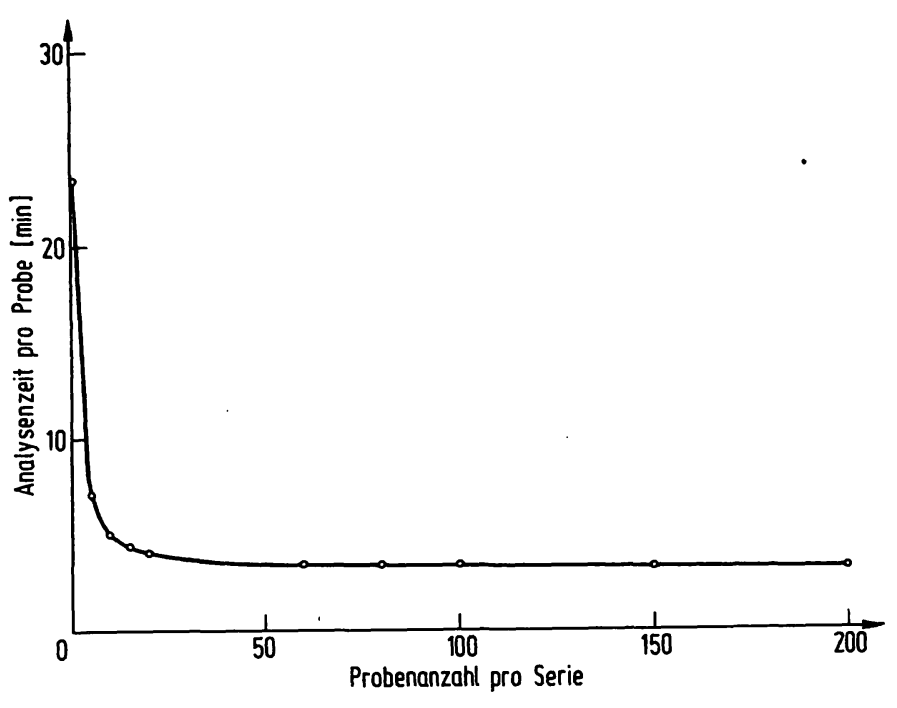

Abb. 1. Analysenzeit pro Probe in Abhängigkeit von der jeweiligen Serienlänge für die manuelle Glucosebestimmung mit der Boehringer Testcombination Nr. 15931.

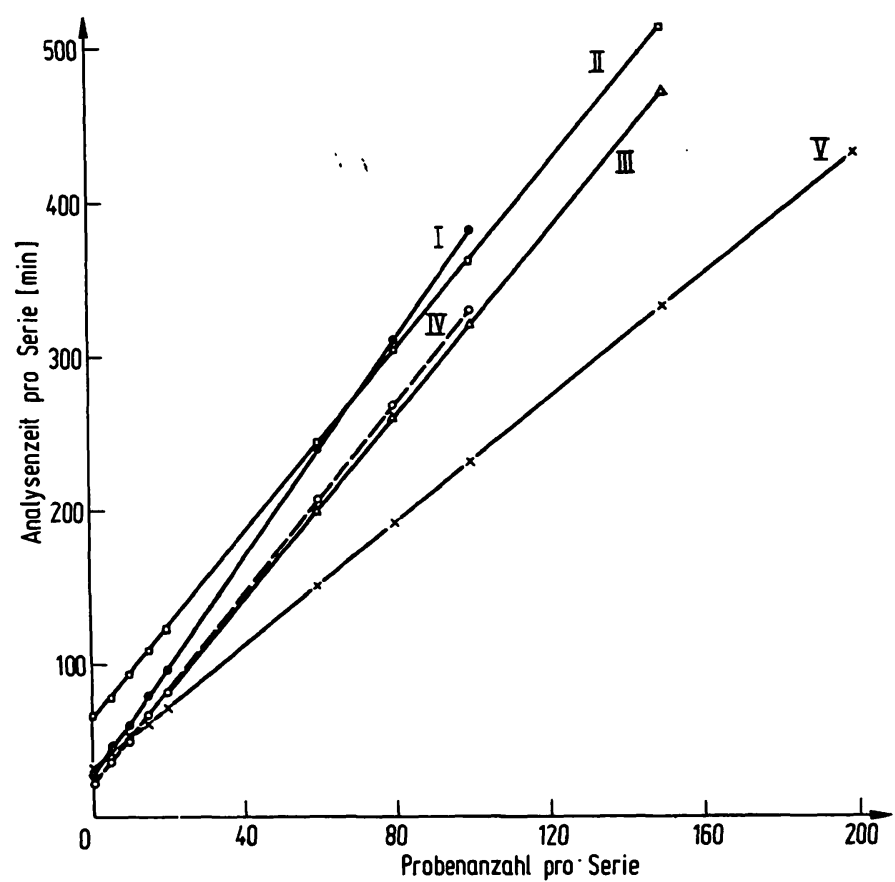

Abb. 2. Analysenzeit pro Serie für verschiedene manuelle Methoden mit Boehringer Testcombinationen. Photometer: Typ 1101 von Eppendorf Gerätebau $\mathrm{GmbH}$ mit Halbmikro-Absaugküvetten. I. Creatinin, Nr. 15 943, II. Harnsäure Nr. 15865, III. Harnstoff Nr. 15930, IV. Glucose Nr. 15931, V. Protein Nr. 15899.

Das in Tabelle 1 dargestellte Verfahren entspricht einem ähnlichen Vorschlag von Bechtler (2), der die Gesamtkosten jedoch auf die Einzelanalyse bezieht. Dieser Autor setzt eine fixe Zeit pro Probe ein, die sich ergibt, wenn während 400 Minuten analysiert wird. Dieses Vorgehen führt nach unseren Erfahrungen nicht immer zu einem Schätzwert für die kritische Serienlänge. Die in Abbildung 1 dargestellte Kurve läßt sich linearisieren, wenn die Einzelanalysenzeit mit n multipliziert wird, d. h. die für die ganze Serie erforderliche Zeit in Abhängigkeit von der Probenanzahl aufgetragen wird
(Abb. 2). Bei allen von uns untersuchten Verfahren (Abb. 2-5) zeigt sich ein linearer Anstieg der seriellen Analysenzeit mit zunehmender Probenanzahl, der gelegentlich stufenförmig erfolgt (Abb. 3).

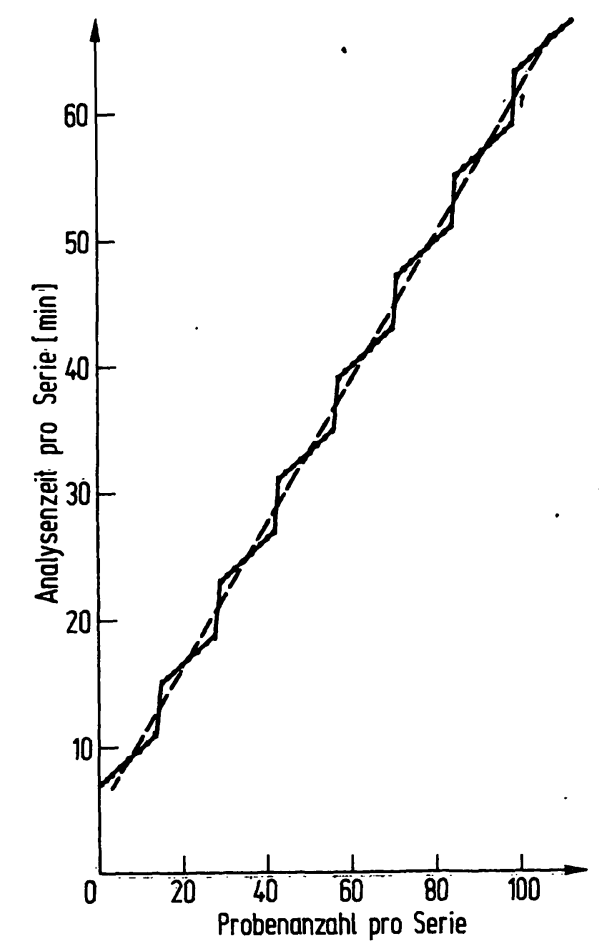

Abb. 3. Analysenzeit pro Serie für die Glucosebèstimmung mit einem GEMSAEC Gerät. Die experimentellen Bedingungen entsprechen den Angaben bei (5).

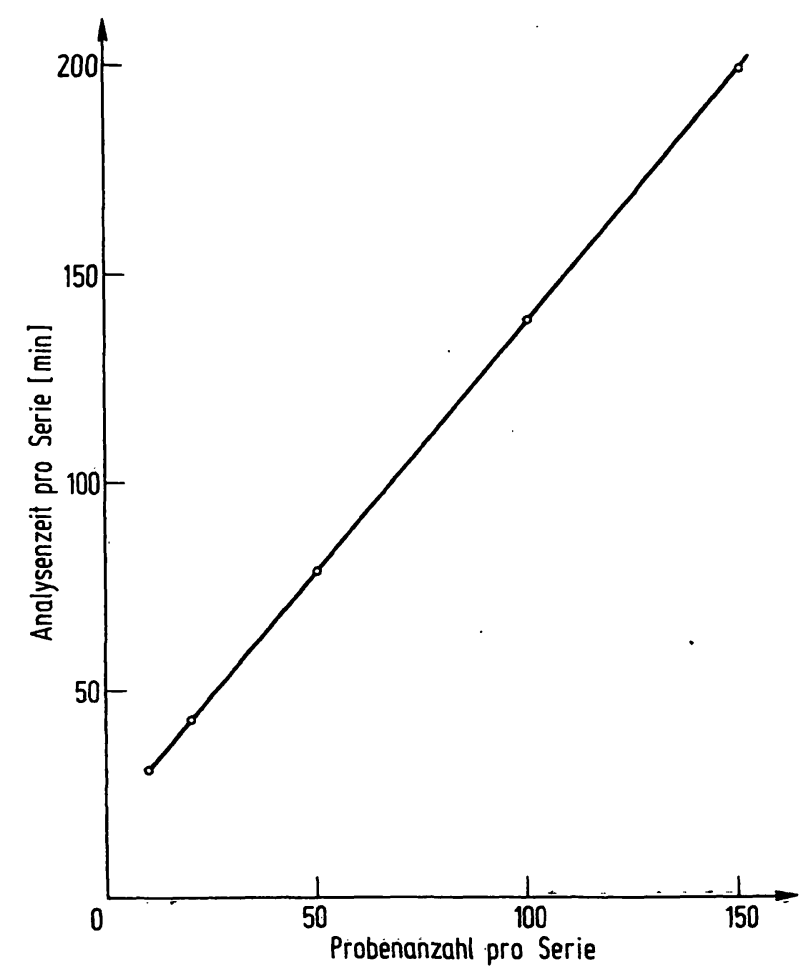

Abb. 4. Analysenzieit pro Serie mit einem Mehrkanalgerät (SMA 6/60, Technicon GmbH). Die Bedienung des Gerrätes erfolgt nach Angaben des Herstellers.

Probenfolge:

5 Leerwerte $\left(\mathrm{H}_{2} \mathrm{O}\right), 3$ Referrenz-, 2 Kontroll-, $10 \mathrm{~Pa}$ tienten-, 2 Referenz-, 10 Patientenproben, etc. 
Die Serienanalysenzeit setzt sich bei den manuellen Bezugsmethoden im allgemeinen aus folgenden Komponenten zusammen:

1. Vorbereitung des Testansatzes (Pipettieren von Proben und Reagenzien, Mischen etc.)

2. Zentrifugation (Enteiweißung etc.)

3. Evtl. Wiederholung von 1.

4. Inkubation

5. Meßvorgang und Auswertung (bei Enzymbestimmungen mit Küvettenwechselautomatik 7,0:6 Minuten/Probe; bei Endwertmethoden mit Probenleerwert 2,0 Minuten und ohne Leerwert 1,5 Minuten/ Probe).

Die Analysenzeit enthält dic Zeiten, die für das Mitführen einer Kontrollprobe und gegebenenfalls von Standard- und Leerwert erforderlich sind. Alle Serien werden bei unseren Berechnungen in-toto aufbereitet.

Die Zeit für die Arbeitsplatzvorbereitung (Ansetzen von Reagenzien, Vorsortieren der Proben etc.) wird bei diesen Betrachtungen nicht berücksichtigt, da hier keine wesentlichen Unterschiede zwischen den meisten manuellen und mechanisierten Verfahren bestehen. Die Clinical Laboratory Study des Chicago Hospital Council (3) berücksichtigt ebenfalls eine Abhängigkeit der Analysenzeit von der Serienlänge. Auch bei diesen Daten scheint eine lineare Beziehung zwischen der seriellen Analysenzeit $(t \cdot n)$ und der Probenanzahl
Tab. 2. Berechnung der Personalkosten

\begin{tabular}{llll}
\hline BAT-Gruppe & $\begin{array}{l}\text { Bruttogehalt }^{\mathrm{a}} \\
\text { (DM) }\end{array}$ & \multicolumn{2}{l}{$\begin{array}{l}\text { Arbeitszeit/Monat } \\
\text { I972 (Stunden) }\end{array}$} \\
\hline VIII & 1294,48 & Januar & 176,5 \\
VI b & 1777,91 & Februar & 176,5 \\
VII & 1309,22 & März & 185 \\
VI b & 1500,31 & April & 159,5 \\
VII & 1299,33 & Mai & 168 \\
IV b & $1948,-$ & Juni & 168 \\
VI b & $1390,-$ & Juli & 176,5 \\
V b & 1814,63 & August & 193,5 \\
IV b & 2068,37 & Scptember & 168 \\
Mittelwert & 1600,25 & Oktober & 185 \\
& & Novembur & 168 \\
& & Dezember & 151 \\
& & Mittelwert & 173 \\
\hline
\end{tabular}

Abwesenheit

(Beurlaubung, Krankhcit, otc.): 3 Monatc

Arbeitsstunden/MTA im Jahr 1972: $173 \cdot 9=1557$ Stunden

Ausgaben/MTA im Jahr 1972: $\quad 1600,25 \cdot 13=20.803,25 \mathrm{DM}$

1 MTA-Arbeitsstunde kostet: $\quad 20.803,25: 1557=13,36 \mathrm{DM}$

1 MTA-Minute kostet: $0,22 \mathrm{DM}$

a) letztes Viertel 1972, cinschlicßlich aller Arbcitgebcranteilc b) Effektive Arbcitszeit: 42 Stunden pro Wochc

zu bestehen; jedoch steigt die Analysenzeil steiler an (z. B. $t \sim 4,8 n+12,5$ für die Aktivitätsbestimmung der Aspartat-Aminotransferase, Manual Method-B $+L$ Spectrophotometer) als bei unseren Werten (Tab. 3

Tab. 3. Die kritische Serienlänge für dic enzymatische Glucosebestimmung mit verschicdenen Analysensystemen

\begin{tabular}{|c|c|c|c|c|c|}
\hline Methode & & $\begin{array}{l}\text { Manuell }{ }^{1} \text { ) } \\
\text { ( }\end{array}$ & $\begin{array}{l}\text { Eppendorf } 5090^{2} \text { ) } \\
\text { II }\end{array}$ & $\begin{array}{l}\text { GEMSAEC }{ }^{3} \text { ) } \\
\text { III }\end{array}$ & $\begin{array}{l}\text { Centrifichem }{ }^{4} \text { ) } \\
\text { IV, V }\end{array}$ \\
\hline \multicolumn{6}{|l|}{ A) Fixe Kosten: } \\
\hline $\begin{array}{l}\text { I. Gerätekosten }{ }^{5} \text { ) } \\
\text { II. } 1,5 \% \text { von I }\end{array}$ & $\begin{array}{l}\text { (DM) } \\
(\mathrm{DM})\end{array}$ & $\begin{array}{r}8.600,60 \\
129,01\end{array}$ & $\begin{array}{r}32.655,45 \\
489,83\end{array}$ & $\begin{array}{r}166.659,25 \\
2.499,89\end{array}$ & $\begin{array}{r}153.515,- \\
2.302 .73\end{array}$ \\
\hline III. Glaswaren & (DM) & $216,-$ & - & - & - \\
\hline $\begin{array}{l}\text { IV. Einmalmaterial pro Serie } \\
\text { V. Reagenzienkosten/Serie }\end{array}$ & $\begin{array}{l}\text { (DM) } \\
(\mathrm{DM})\end{array}$ & $\begin{array}{l}0,87 \\
0,54\end{array}$ & $\begin{array}{l}0,68 \\
1,26\end{array}$ & 0,37 & 0,50 \\
\hline$K_{f}=(I+I I \times 5): 1800+\frac{I I I}{100}+I$ & $\mathrm{IV}+\mathrm{V}(\mathrm{I}$ & DM) 8,71 & 21,44 & 99,90 & 92,18 \\
\hline \multicolumn{6}{|l|}{ B) Variable Kosten: } \\
\hline $\begin{array}{l}\text { I. Einmalmaterial }\left(E_{p}\right) \\
\text { pro Probe } \\
\text { II. Reagenzienkosten }\left(R_{p}\right) \\
\text { III. Analysenzeit } t_{1} \\
\quad t_{2}\end{array}$ & $\begin{array}{l}(\mathrm{DM}) \\
(\mathrm{DM}) \\
(\min ) \\
(\min )\end{array}$ & $\begin{array}{c}0,31 \\
0,18 \\
\left.52,5(10 \text { Proben })^{7}\right) \\
\left.285,5(100 \text { Proben })^{7}\right)\end{array}$ & $\begin{array}{c}0,24 \\
0,18 \\
\left.45(10 \text { Proben })^{8}\right) \\
180\left(100 \text { Proben }^{8}\right)\end{array}$ & $\begin{array}{c}0,14 \\
\left.0,22^{6}\right) \\
\left.11(13 \text { Proben })^{9}\right) \\
83\left(130 \text { Proben }^{9}\right)\end{array}$ & $\begin{array}{c}0,05 \\
\left.0,60(0,10)^{6}\right) \\
\left.10(27 \text { Proben })^{9}\right) \\
\left.58(189 \text { Proben })^{9}\right)\end{array}$ \\
\hline \multicolumn{3}{|l|}{ C) $\left.S_{k}^{10}\right)$} & 44 & 153 & $230(93)$ \\
\hline
\end{tabular}

1) Eppendorf Photometer 1101, 2 Absaugpumpen 3500, 1 Eppendorf Zentrifuge 3200, 3 Eppendorf Pipctten.

2) Eppendorf Digitalmeßplatz 5090, 1 Zentrifuge 5411, 1 Rotationsmischer 5431, 1 Eppendorf Dosicrer, 1 Eppendorf Probe-ReagenzDosierer.

3) Gemsaec Analysenautomat, 1 Eppendorf Zentrifuge 5411, 1 Eppendorf Pipette: Methodik siehe (6).

4) Methodik und Reagenzienkosten nach Angaben von Hoffmann-La Roche (In Klammern: Prcisc für Reagenzien von BoehringcrMannheim).

5) Kosten in DM.

6) Die Reagenzienkosten für je 1 Leerwert, Standard und Kontrolle werden auf cine Rotorladung (13 bzw. 27 Proben) umgelegt. Jede Serie enthält 1 Leerwert, 1 Standard und 1 Kontrollprobe.

7) Es werden zunächst 12 Proben zentrifugiert, die restlichen Proben während der Messung.

8) Jede Serie enthält 5 Leerwerte, 1 Standard und 1 Kontrollprobe.

9 ) Jede Rotorladung enthält 1 Leerwert, 1 Standard und 1 Kontrollprobc.

10) Rechnerisch nach Formel 4 ermittelt. 
I : $t=2,0 n+5,0)$, da Proben- und Arbeitsplatzvorbereitung mit einbezogen wurden. Zieht man diese Zeit $a b$, sind die in umfangreichen Studien ermittelten Analysenzeiten (3) trotzdem länger als die von uns verwendeten, da noch Wegezeiten (innerhalb des Labors) berïcksichtigt werden. Wir gehen davon aus, daß der Arbeitsplatz optimal eingerichtet wird und keine zusätzlichen Wege erforderlich sind (Tab. 3). Auch bei steigender Serienlänge werden alle Proben parallel (batch-weise) Schritt für Schritt aufbereitet, ohne daß verschiedene kleinere Unterserien ineinandergeschachtelt werden. Betriebsausfälle werden ebenfalls nicht berücksichtigt. Daher ist die kritische Serienlänge ein Wert, der bei optimalen Bedingungen gilt. Sie muß bei ungenügender Wartung und Bedienung des Gerätes zwangsläufig höher liegen.

Die Gesamtkosten einer Analysenserie steigen ebenfalls linear mit der Probenanzahl bei allen bisher unter-

suchten Fällen an (Abb. 5). Daher kann das in Tabelle 1 beschriebene, zeitaufwendige Verfahren vereinfacht werden: Die Funktion der Gesamtkosten einer Analysenserie lautet nach Tabelle 1

$$
K_{t}=K_{f}+R_{p} \cdot n+E_{p} \cdot n+P \cdot t_{n}
$$

Wie oben gezeigt besteht eine lineare Beźiehung zwischen $t_{n}$ und $n$ :

$$
t_{n}=a \cdot n+b
$$

Bei der kritischen Serienlänge $\left(n=S_{k}\right)$ wird $K_{t A}=K_{t B}$ und somit

$$
S_{k}=\frac{\left(K_{f B}-K_{f A}\right)+P\left(b_{B}-b_{A}\right)}{\left(R_{p A}-R_{p B}\right)+\left(E_{p A}-E_{p B}\right)+P\left(a_{A}-a_{B}\right)}
$$

Ermittelt man die Analysenzeit $t_{1}$ und $t_{2}$ für 2 Serienlängen, kann man $a$ und $b$ bestimmen und erhält

$$
S_{k}=\frac{\left(K_{f B}-K_{f A}\right)+P\left(\frac{t_{B 1} \cdot n_{B 2}-t_{B 2} \cdot n_{B 1}}{n_{B 2}-n_{B 1}}-\frac{t_{A 1} \cdot n_{A 2}-t_{A 2} \cdot n_{A 1}}{n_{A 2}-n_{A 1}}\right)}{\left(R_{p A}-R_{p B}\right)+\left(E_{p A}-E_{p B}\right)+P \cdot\left(\frac{t_{A 2}-t_{A 1}}{n_{A 2}-n_{A 1}}-\frac{t_{B 2}-t_{B 1}}{n_{B 2}-n_{B 1}}\right)}
$$

Zur Berechnung der kritischen Serienlänge werden von einer Methode also folgende Werte benötigt:

1. Fixe Kosten $\left(\mathrm{K}_{\mathrm{f}}\right)$

2. Reagenzienkosten pro Analyse $\left(R_{p}\right)$

3. Kosten für Einmalmaterial pro Analyse $\left(E_{p}\right)$
4. Analysenzeit für $n_{1}$ Proben $\left(t_{1}\right)$

5. Analysenzeit für $n_{2}$ Proben $\left(t_{2}\right)$

Da die Analysenzeit bei manchen Verfahren mit der Probenanzahl stufenförmig ansteigt, sollte $n_{1}$ und $n_{2}$ nicht zu nahe beieinander liegen. Wir wählen $n_{1}=10$, und $n_{2}=100$, so daß sich Gleichung (4) vereinfacht nach

$$
S_{k}=\frac{\left(K_{f B}-K_{f A}\right)+P \cdot 1,11\left(t_{B 1}-t_{A 1}\right)+P \cdot 0,11\left(t_{A 2}-t_{B 2}\right)}{\left(R_{p A}-R_{p B}\right)+\left(E_{p A}-E_{p B}\right)+P \cdot 0,01\left(t_{A 2}-t_{A 1}-t_{B 2}+t_{B 1}\right)}
$$

Bei mechanisierten Verfahren bieten sich für $t_{1}$ gelegentlich auch größere Serienlängen an (z. B. Tabelle 3); in solchen Fällen kann man Gleichung (4) verwenden.

Der in Abbildung 3 dargestellte stufenförmige Verlauf der Analysenzeit läßt sich am besten durch die Gerade $t=0,58 \cdot n+5,0$ beschreiben. Die durch $t_{n=i}$ und $t_{n=100}$ gelegte Gerade, hat die Funktion $t^{\prime}=0,58$. $n+6,5$. Setzt man $t$ und $t^{\prime}$ in Gleichung (3) ein und berechnet die kritische Serienlänge für die Glucosebestimmung mit dem GEMSAEC in bezug auf eine manuelle Methode (Abb. 2, VI), entsprechend der Daten in den Abbildungen 2, 3 und 5, wird $n=202$ für $S_{k}$ und $n=201$ für $S_{k^{\prime}}$. Die Lage von $t_{1}$ ist hier also nicht kritisch für die Berechnung von $S_{k}$.

Die Analysenzeit für die Glucosebestimmung an einem Centrifichem-Gerät $(t=0,3 n+2,0)$ steigt mit der Serienlänge weniger steil an als beim GEMSAEC (Abb. 3: $t=0,58 n+5,0)$. Trotzdem ist die kritische Serienlänge länger, wenn man das von der europäischen Vertriebsfirma Hoffmann-La Roche empfohlene Ver- fahren, sowie den Preis für deren Reagenzien einsetzt. Verwendet man die Testcombination Nr. 15931 von Boehringer-Mannheim, sowohl für die manuelle Bezugsals auch für die Centrifichem-Methode, verkürzt sich die kritische Serienlänge erheblich (Abb. 5).

Die Steilheit der linearen Funktion zwischen Gesamtkosten und Serienlängen (wie z. B. in Abb. 5) wird durch die variablen Kosten $\left(R_{f}, E_{f}, P\right.$, Probenfrequenz), der Schnittpunkt mit der Ordinate von den fixen Kosten einer Methode bestimmt. Je niedriger die fixen und variablen Kosten und je höher die mögliche Probenfrequenz eines mechanisierten Verfahrens sind, desto kleiner ist der Schätzwert für die kritische Serienlänge.

In den letżten Jahren sind vor allem die Personalkosten gestiegen, während die Sachkosten weitgehend konstant geblieben sind. Diese Entwicklung führt zu einer Verkürzung der kritischen Serienlängen. In Abbildung 8 ist dieser Effekt am Beispiel der Aspartat-Aminotransferase-Bestimmung mit einem GEMSAEC-Gerät gezeigt. 


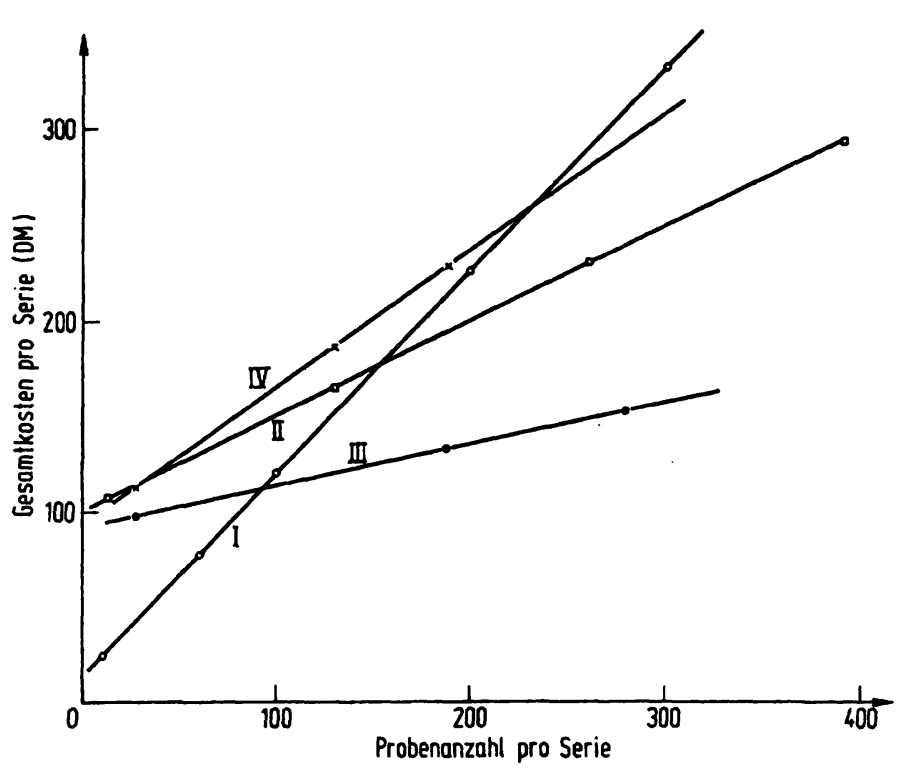

Abb. 5. Die Gesamtkosten der enzymatischen Glucosebestimmung pro Serie mit einem GEMSAEC (II) und einem Centrifichem (III, IV) Analysengerät im Vergleich mit einem manuellen Verfahren (I). Bei IV wurden die Preise der Reagenzien von Hoffmann-La Roche, bei I-III die von Boehringer Mannheim eingesetzt. Den Berechnungen wurden die Daten in Tabelle 3 zugrunde gelegt.

\section{Die kritische Serienlänge}

\section{a) für die Verwendung von Glasküvetten}

Eine Künststoffküvette kostet etwa 0,07 DM. Normalküvetten aus Glas (13,20 DM) müssen nach jeder Messung gespült werden. Dadurch benötigt man bei der heute meist verwendeten 6 fach-Küvettenwechselautomatik mindestens $2 \times 6$ Einzelküvetten. Setzt man zur Reinigung (spülen und föhnen) einer Küvette eine durchschnittliche Minimalzeit von 15 Sekunden an, so ergibt sich eine kritische Serienlänge von etwa 115 (Abb. 6). Dabei ist nicht die in regelmäßigen Abständen erforderliche gründliche Spülung mit Detergentien, Chromschwefelsäure oder dgl. berücksichtigt. Vom Standpunkt der Wirtschaftlichkeit lohnt sich daher die Verwendung von Glasküvetten nicht mehr.

\section{b) für Einkanalgeräte}

Die kritische Serienlänge nimmt mit den Investitionskosten für den Ausbau eines Analysensystems in Richtung Vollmechanisierung zu, wie wir am Beispiel der Glucosebestimmung mit Geräten der Firma Eppendorf gezeigt haben (1). Âhnlịch läßßt sich durch Berechnung der kritischen Serienlänge zeigen, daß sich der Ausbau eines Analog-Meßplatzes (mit Küvettenwechselautomatik und Schreiber) zu einem Enzymautomat 5010 von Eppendorf erst lohnt, wenn etwa 130 Proben für eine tägliche Serie anfallen (Tạb. 4). Die weitere Ausstattung desselben Meßplatzes mit Rechner und Drucker zur Ausgabe des Endresultates in Enzymeinheiten, wird erst ab 170 Proben in bezug auf das teilmechanisierte Verfahren ( $I$ in Tab. 4) rentabel. Diese nach Formel

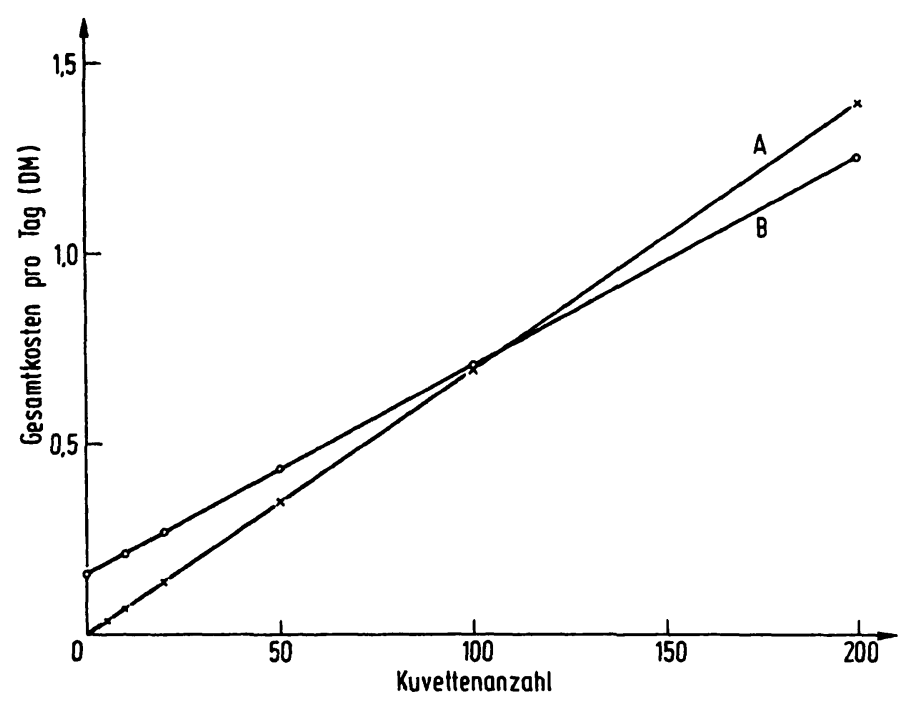

Abb. 6. Die kritische Serienlänge für die Verwendung von Glasküvetten (B) im Vergleich zu Einmal-Küvetten aus Kunststoff (A). Da meistens mit Wechselau tomatik gearbeitet wird, sind mindestens $2 \times 6$ Glasküvetten (à 13,20 DM) erforderlich. Für die Abnutzung eines Föhns, wurden 0,02 DM pro Tag und für die Spülzeit pro Küvette etwa 15 Sekunden geschätzt.

(4) berechneten Zahlen stimmen gut mit graphisch ermittelten (entsprechend Abb. 6) $\mathrm{S}_{\mathrm{k}}$-Werten bei der Bestimmung der Aspartat-Aminotransferase überein (Abb. 7). Auch hier verläuft die Beziehung zwischen den Gesamtkosten und der Probenzahl einer Serie linear.

Die kritische Serienlänge für die Aktivitätsbestimmung der Transaminasen mit einem GEMSAEC-Gerät beträgt etwa 170 Proben. Bei Bezug auf ein manuelles Verfahren mit Küvettenwechselautomatik und ungefähr 170 Proben bei Bezug auf ein Eppendorf-Gerät 5010 mit Drucker (Abb. 7).

Analysenautomaten nach dem Küvettenrotorverfahren sind z. Zt. Einkanalgeräte, die für ein rasches Umrüsten geeignet sind. Daher werden die meisten Anwender mehrere Methoden nacheinander täglich einsetzen. In diesem Fall kann man wie bei einem Mehrkanalgerät so vorgehen, daß man die entsprechenden fixen Kosten nur bei der ersten Methode berücksichtigt. Zur groben Schätzung genügt in der Praxis aber auch, eine Methode mit mittlerem Kostenaufwand auszuwählen und deren Wert für die kritische Serienlänge um etwa $20 \%$ zu erhöhen. So erhält man einen Hinweis, wieviele Analysen verschiedener Methoden mindestens pro Tag mit einem solchen Gerät anfallen sollten.

\section{c) für Mehrkanalgeräte}

Die kritische Serienlänge wurde für ein Mehrkanalgerät am Beispiel eines SMA 6/60 von Technicon unter zwei Betriebsmodalitäten geschätzt:

1. Alle 6 Bestimmungen sind angefordert und

2. 1, 2, 3 oder 4 Methoden sind nicht indiziert. 


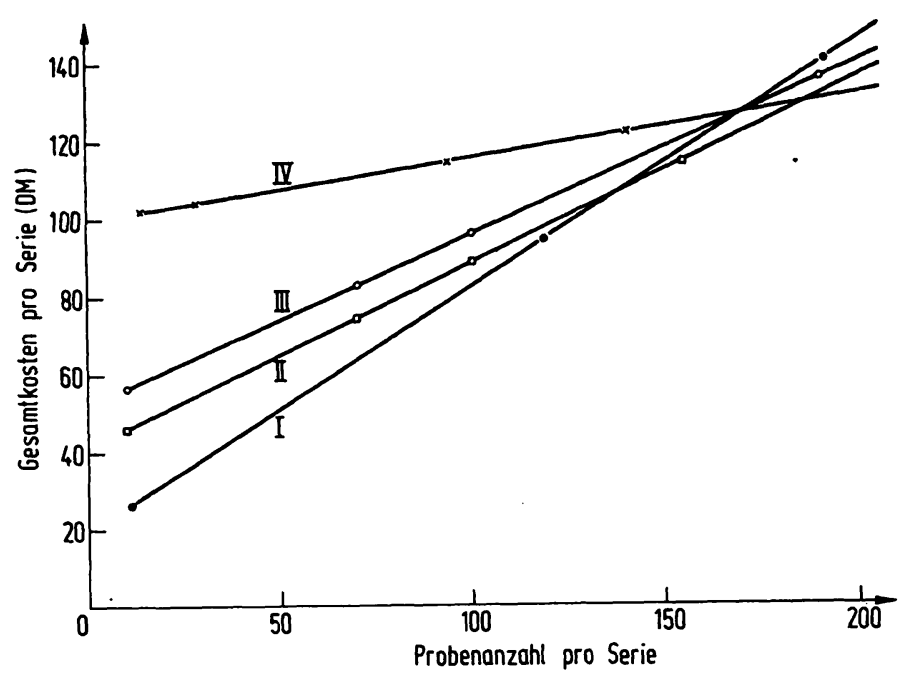

Abb. 7. Die graphische Ermittlung der kritischen Serienlänge für die Aktivitätsbestimmung der Serum-Aspartat-Aminotransferase (optimierter Test) entsprechend (4). Die verwendeten Daten entsprechen den Angaben in der Tabelle 4.

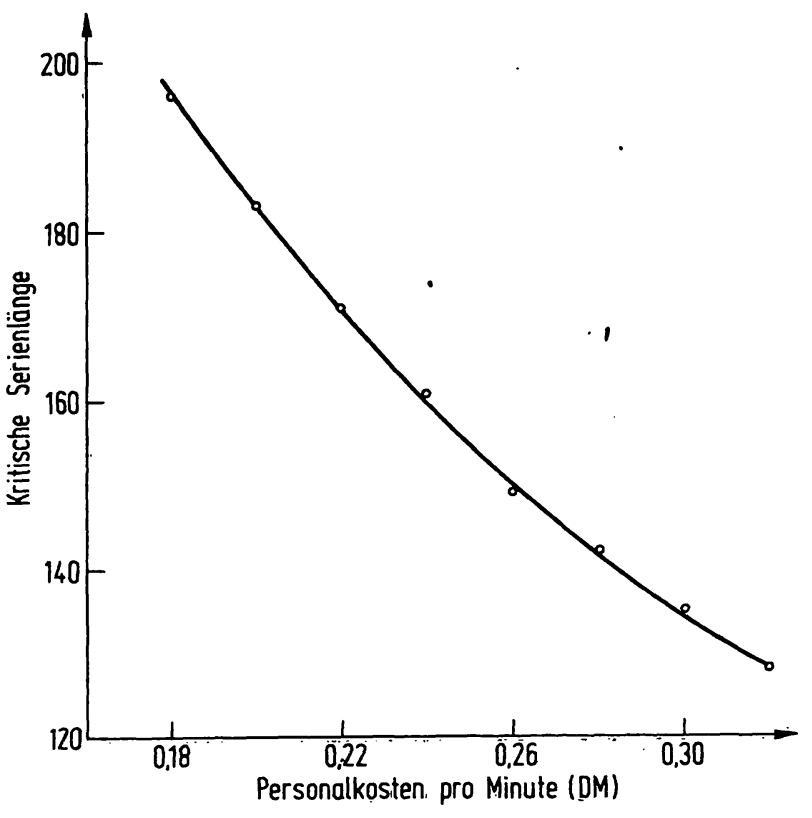

Abb: 8. Der Einfluß der Personalkosten auf die kritische Serienlänge für die Aktivitätsbestimmung der Serum-AspartatAminotransferașe mit einem GEMSAEC-Gerät. Das manuelle Bezugsverfahren verwendet einen Digital-Meßplatz 5085 von Eppendorf. Den Berechnungen sind die Daten in Tabelle 4 zugrunde gelegt.

Tab. 4. Die kritische Serienlänge bei der Bestimmung der Aspartat-Aminotransferase-Aktivität in Serumproben mit verschiedenen mechanisierten Analysensystemen

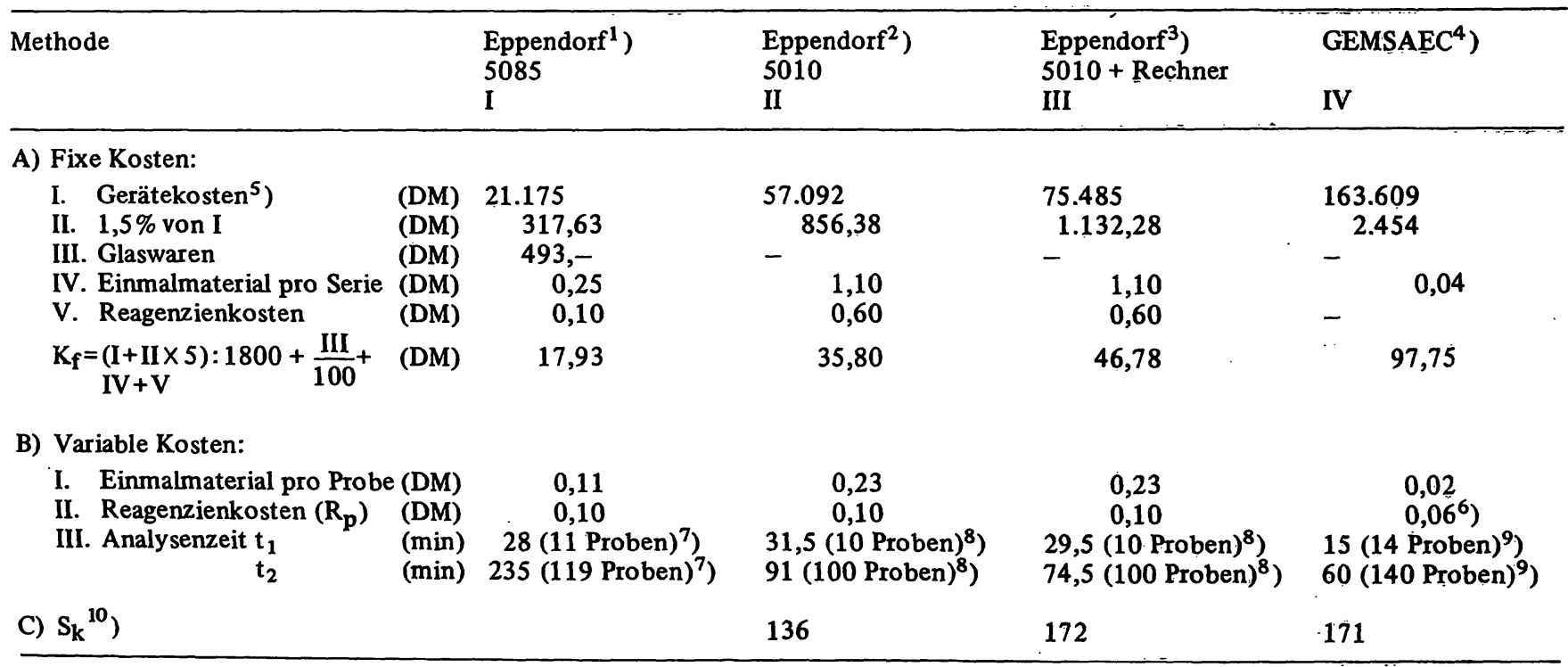

1) Analogmeßplatz Eppendorf 5085 mit Haake Thermostat u. 3 Eppendorf Pipetten;

2) Analysenautomat Eppendorf 5010 und 1 Eppendorf Pipette.

3) Analy senautomat Eppendorf 5010 mit Rechner + Drucker + einer Eppendorf Pipette.

$\left.\left.{ }^{1}\right)^{2}\right){ }^{3}{ }^{3}$ ) Bedienung der Geräte nach Vorschrift des Herstellers. Reaktionslösung: $500 \mu 1$, Serum: $100 \mu 1,2-0 x o g l u t a r a t: 50 \mu 1$, Merckotest 3375. Die Reaktionsbedingungen entsprechen den Empfehlungen der Deutschen Gesellschaft für Klinische Chemie (4). Auswertezeit bei ${ }^{1}$ ) u. ${ }^{2}$ ) 10 s pro Probe.

4) Analy senautomat GEMSAEC; Rotoloader $5 \mathrm{~min}$, Vorinkubation $5 \mathrm{~min}$ bei $25^{\circ} \mathrm{C}$, Zentrifugenlauf $4 \mathrm{~min}$, Gesamtzeit des 1. Laufes $15 \mathrm{~min}$, der folgenden Läufe je $5 \mathrm{~min} ; 1$. Reading nach $10 \mathrm{~s}$ dann $5 \times$ alle $30 \mathrm{~s}$, pro Lauf 1 Kontrollprobe.

5) Alle Kosten in DM

6) einschl. Reagenzienkosten für 1 Leerwert u. 1 Kontrollprobe pro Rotorladung (14 Proben).

7) Die Analysenzeit gilt für die angegebene Probenanzahl + 1 Kontrollprobe, entsprechend bei

8) +5 Leerwerte u. 1 Kontrollprobe u. bei

9 ) 1 Leerwert und 1 Kontrollprobe pro Rotorladung.

10) Kritische Serienlänge (Probenanzahl), berechnet nach Formel (4). 
Die Wirtschaftlichkeit der zweiten Betriebsart wird in der Praxis bei indiskriminiert funktionierenden Analysensystemen immer wieder in Frage gestellt. Für die erstere Modalität errechnet sich eine kritische Serienlänge von 34 Proben (Tab. 5).

Als manuelle Bezugsmethoden wurden solche eingesetzt, die entweder bei uns in der Routine verwendet werden oder als fertige Testcombinationen von Boehringer Mannheim erhältlich sind. Die Reagenzien der Bezugsmethoden wurden addiert (Tab. 5). Bei der Analysenzeit wurden die Elektrolyte nicht mit berücksichtigt, da diese während der Inkubationszeiten der anderen manuellen Methoden (z. B. 30 Minuten bei der Proteinbestimmung) durchgeführt werden können. Unter den fixen Kosten wurden die Anschaffungskosten für jeweils 1 Flammenphotometer und 1 Eppendorf
Photometer 1101 in die Summe der manuellen Bezugsmethoden eingesetzt.

Wird in dem von uns gewählten Beispiel die Glucosebestimmung in einer Serie umsonst, d. h. nicht indiziert durchgeführt, erhöht sich die kritische Serienlänge auf 61 Proben. Auch wenn nur 3 Bestimmungen für eine Probe angefordert werden, ist der Betrieb eines SMA $6 / 60$ bei einer täglichen Serie von mehr als 230 Proben wirtschaftlich (Tab. 6). Die alleinige Messung der Natriumund Kaliumkonzentration lohnt sich in diesem System nicht. Diese Daten zeigen, daß ein SMA 6/60 Gerät auch bei nur teilweiser Auslastung der einzelnen Kanäle noch wirtschaftlich sein kann.

Es handelt sich bei den vorliegenden Investitionsrechnungen um ein statisches Verfahren, das auf dem Prinzip der Kostenvergleichsrechnung ( 7 ) beruht. Zinsen für

Tab. 5. Die kritische Serienlänge eines SMA 6/60 Gerātes in bezug auf 6 manuelle Methoden

\begin{tabular}{|c|c|c|c|c|c|c|c|c|}
\hline Methode & & $\left.\mathrm{Na}, \mathbf{K}^{\mathbf{1}}\right)$ & Phosphor $^{2}$ ) & Protein ${ }^{3}$ ) & Harnstoff & Glucose $^{5}$ ) & $\begin{array}{l}\text { Summe }{ }^{6} \text { ) } \\
\text { der manu- } \\
\text { ellen Be- } \\
\text { stimmungen }\end{array}$ & SMA $6 / 60^{7}$ ) \\
\hline A) Erforderliche Geräte: ${ }^{8}$ ) & & $a, b$ & $c, 5 \times f$ & $c, 3 \times f$ & $c, d, 2 \times f$ & $c, e, 4 \times f$ & $\begin{array}{l}a, b, c, d, e, \\
14 \times f\end{array}$ & g \\
\hline \multicolumn{9}{|l|}{ B) Fixe Kosten: } \\
\hline $\begin{array}{l}\text { I. Gerätekosten }{ }^{9} \text { ) } \\
\text { II. } 1,5 \% \text { von I } \\
\text { III. Glaswaren }{ }^{10} \text { ) } \\
\text { IV. Einmalmaterial pro }\end{array}$ & $\begin{array}{l}(\mathrm{DM}) \\
(\mathrm{DM}) \\
(\mathrm{DM})\end{array}$ & $\begin{array}{r}11.709,- \\
175,64 \\
-\quad\end{array}$ & $\begin{array}{c}7.655,- \\
114,82 \\
216,-\end{array}$ & $\begin{array}{l}7.471,- \\
112,06 \\
216,-\end{array}$ & $\begin{array}{r}8.092,- \\
121,37 \\
219,60\end{array}$ & $\begin{array}{l}8.473,- \\
127,10 \\
216,-\end{array}$ & $\begin{array}{r}21.930,- \\
328,95 \\
219,60\end{array}$ & $\begin{array}{r}214.873,- \\
3.223,10 \\
5,86\end{array}$ \\
\hline $\begin{array}{l}\text { Serie }{ }^{11} \text { ) } \\
\text { V. Reagenzienkosten }\end{array}$ & (DM) & 0,12 & 0,40 & 0,51 & 0,30 & 0,51 & 1,84 & 0,63 \\
\hline $\begin{array}{c}\left.\text { pro Serie }{ }^{11}\right) \\
K_{f}=(I+I(\times 5): 1800+\end{array}$ & (DM) & 0,03 & 0,24 & 0,04 & 0,60 & 0,81 & 1,72 & 5,71 \\
\hline$\left.\frac{\mathrm{III}}{100}+\mathrm{IV}+\mathrm{V}^{12}\right)$ & (DM) & 7,14 & 7,37 & 7,17 & 7,93 & 8,54 & 18,85 & 140,53 \\
\hline \multicolumn{9}{|l|}{ C) Variable Kosten: } \\
\hline $\begin{array}{l}\text { I. Einmalmaterial (E) } \\
\text { pro Probe }^{13} \text { ) } \\
\text { II. Reagenzienkosten }\end{array}$ & (DM) & 0,04 & 0,11 & 0,15 & 0,10 & 0,22 & 0,62 & 0,09 \\
\hline $\begin{array}{l}(\mathrm{R})^{[3} \text { ) } \\
\text { III. Analysenzeit }\end{array}$ & (DM) & 0,01 & 0,08 & 0,02 & 0,20 & 0,27 & 0,58 & 0,38 \\
\hline $\begin{array}{l}t_{1}(10 \text { Proben }) \\
t_{2}(100 \text { Proben })\end{array}$ & $\begin{array}{l}(\min ) \\
(\min )\end{array}$ & $\begin{array}{l}10 \\
78\end{array}$ & $\begin{array}{r}54 \\
324\end{array}$ & $\begin{array}{r}66 \\
336\end{array}$ & $\begin{array}{r}60 \\
285\end{array}$ & $\begin{array}{r}61 \\
286\end{array}$ & $\begin{array}{r}241 \\
1.231\end{array}$ & $\begin{array}{r}31 \\
139\end{array}$ \\
\hline D) $\left.S_{k}{ }^{14}\right)$ & & & & & & & & 34 \\
\hline
\end{tabular}

$\left.{ }^{1}\right)$ IL Flammenphotometer 143 (a) Methodik nach Vorschrift der Herstellerfirma

2) Anorganischer Phosphor Roche Diagnostica

3) Weichselbaum, T. E. (1946), Amer. J. Clin. Path. 10,40; Richterich, R. Klin. Chemie, 2. Auflage 1968, Reagenzien von E. Merck

${ }^{4}$ ) Merckotest Bestell-Nr. 3334

5) GOD-Perid-Methode Boehringer Bestell-Nr. 15755

6) Zusammenfassung der manuellen Methoden, der Kaufpreis für (c) wurde nur einmal eingesetzt, die Analysenzeit für Na und K nicht berücksichtigt.

7) Bedienung des Gerätes nach Vorschrift des Herstellers, Reagenzienkosten: Technicon Preisliste 1972 (Jahresbedarf, 25\% Skonto). Probenfolge: 5 Leerwerte, 3 Ref., 1 Kontrolle und alle 10 Proben 2 Referenzproben

8) b: DADE-Dilutor, c: Eppendorf Photometer 1101, d: Wasserbad GFL, e: Eppendorf Zentrifuge 3200, f: Eppendorf Pipetten, g: SMA 6/60

9) Gerätepreise von 1972 in DM

10) SMA 6/60: tägl. Kosten für Membranen und Pumpenschläuche (Haltbarkeit: Pumpenschlauch 1 Monat, Membranen 1 Woche)

11) SMA 6/60: einschl. der Kosten für Schreiberpapier, bzw. Referenzserum. Die manuellen Methoden beinhalten 1 Leerwert, 1 Standard und 1 Kontrollprobe.

12) SMA 6/60: $F=(1+I 1 \times 5): 1800+I I I+I V+V$.

13) SMA 6/60: einschl. der Kosten für Einmalmaterial, bzw. Reagenzien für 2 Referenzanaly sen pro 10 Patientenproben.

$\left.{ }^{14}\right)$ Kritische Serienlänge (Probenanzahl) berechnet nach Gleichung (4). 
Tab. o. Die Berechnung der kritischen Serienlänge eines SMA 6/60 Gerätes, wenn einzelne Ergebnisse nicht erforderlich sind. Glucose wird manuell und im SMA 6/60 mit der GOD-Perid Methode bestimmt. Bei I-III ist die Analysenzeit für Na und $\mathrm{K}$ beim manuellen Verfahren berücksichtigt, nicht jedoch bei IV entsprechend dem Vorgehen in Tabelle 5. Alle weiteren Bedingungen und Symbole entsprechen den Angaben in Tabelle 5.

\begin{tabular}{|c|c|c|c|c|c|}
\hline Methode & & $\begin{array}{l}\text { I } \\
\text { Na, K, } \\
\text { Phosphor }\end{array}$ & $\begin{array}{l}\text { II } \\
\mathrm{Na}, \mathrm{K} \\
\text { Glucose }\end{array}$ & $\begin{array}{l}\text { III } \\
\text { Na, K, Phosphor } \\
\text { Protein }\end{array}$ & $\begin{array}{l}\text { IV } \\
\text { Na, K, Phosphor } \\
\text { Protein, Harnstoff }\end{array}$ \\
\hline A) Erforderliche Geräte: & & $a, b, c, 5 \times f$ & $a, b, c, e, 4 \times f$ & $a, b, c, 8 \times f$ & $a, b, c, d, 10 \times f$ \\
\hline \multicolumn{6}{|l|}{ B) Fixe Kosten: } \\
\hline $\begin{array}{l}\text { I. Gerätekosten } \\
\text { II. } 1,5 \% \text { von I } \\
\text { III. Glaswaren } \\
\text { IV. Einmalmaterial pro Serie } \\
\text { V. Reagenzienkosten pro Serie }\end{array}$ & $\begin{array}{l}(\mathrm{DM}) \\
(\mathrm{DM}) \\
(\mathrm{DM}) \\
(\mathrm{DM}) \\
(\mathrm{DM})\end{array}$ & $\begin{array}{r}19.364,- \\
290,46 \\
216,- \\
0,52 \\
0,27\end{array}$ & $\begin{array}{r}20.182,- \\
302,73 \\
216,- \\
0,63 \\
0,84\end{array}$ & $\begin{array}{r}19.697,54 \\
295,46 \\
216,- \\
1,03 \\
0,31\end{array}$ & $\begin{array}{r}20.594,54 \\
308,92 \\
219,60 \\
1,33 \\
0,91\end{array}$ \\
\hline$K_{f}=(I+I I \times 5): 1800+\frac{\text { III }}{100}+I V+V$ & $(\mathrm{DM})$ & 14,52 & 15,68 & 15,62 & 16,73 \\
\hline \multicolumn{6}{|c|}{ C) Variable Kosten: } \\
\hline $\begin{array}{l}\text { I. Einmalmaterial }\left(E_{p}\right) \text { pro Probe } \\
\text { II. Reagenzienkosten }\left(R_{p}\right) \text { pro Probe } \\
\text { III. Analysenzeit } t_{1}(10 \text { Proben }) \\
t_{2}(100 \text { Proben })\end{array}$ & $\begin{array}{l}(\mathrm{DM}) \\
(\mathrm{DM}) \\
(\mathrm{min}) \\
(\mathrm{min})\end{array}$ & $\begin{array}{l}0,15 \\
0,09 \\
64 \\
402\end{array}$ & $\begin{array}{l}0,26 \\
0,28 \\
71 \\
364\end{array}$ & $\begin{array}{l}0,30 \\
0,11 \\
130 \\
738\end{array}$ & $\begin{array}{l}0,40 \\
0,31 \\
180 \\
545\end{array}$ \\
\hline D) $S_{k}$ für SMA $6 / 60$ & & 377 & 232 & 100 & 58 \\
\hline
\end{tabular}

das gebundene Kapital, sowie Einsparungen durch $\mathrm{Ab}$ setzungen für Abnutzung von der Einkommensteuer wurden nicht berücksichtigt; ihre Auswirkungen auf die kritische Serienlänge heben sich teilweise gegenseitig auf und werden in einer folgenden Mitteilung erörtert (in Vorbereitung).

Die kritische Serienlänge soll demjenigen, der sich ein Analysengerät anschaffen möchte, ein Maß für die Rentabilitätsschwelle geben. Sie soll lediglich anzeigen, wie groß der serielle Probenanfall sein muß, damit der Kauf eines größeren Gerätes finanżiell sinnvoll ist. Eine kritische Serienlänge läßt sich für diskriminierende Geräte, die nicht mit einer hohen Analysenfrequenz arbeiten und teuere Reagenzien verwenden, nicht berechnen. Darauf wurde bereits an anderer Stelle (1) hingewiesen.

\section{Danksagung}

Herrn Dr. H. Francke danken wir für wertvolle Anregungen bei der Abfassung des Manuskriptes.

\section{Literatur}

1. Haeckel, R. \& Höpfel (1973), P., Diagnostik 6, 674-78.

2. Bechtler, G. (1970), Biologie Prospective Colleque Pont-àMousson 1, 24-26.

3. Chicago Hospital Council (1965), Clinical Laboratory Study, University Microfilms, High Wycomb, England.

4. Haeckel, R. \& Haeckel, H. (1972), diese Z. 10, 453-61.
5. Standardisierung von Methoden zur Bestimmung von Enzymaktivitäten in biologischen Flüssigkeiten (1972), diese . Z. 10, 182-92.

6. Haeckel, R. (1973) diese Z. 11, 243-48.

7. Bronner, A. (1964), Vereinfachte Wirtschaftlichkeitsrechnung, Beuth-Vertrieb GmbH, Berlin.
Priv. Doz. Dr. R. Haeckel 3000 Hannover Karl-Wiechert-Allee 9 